\title{
Study on the Application of IOT in the Fire Early Warning of Cotton Warehousing
}

\author{
Jia Jiang, Damin Liu, Yibo Jin \\ School of Economics and Management, Hebei University of Science and \\ Technology, Shijiazhuang 050018, China \\ jiang_jia67@163.com
}

\begin{abstract}
In order to achieve the effective early warning of fire in the process of cotton warehousing, the conceptual model of cotton smoldering was constructed based on the existing research results of the formation mechanism of cotton smoldering. Then the front-end monitoring network of cotton warehousing environment was designed on the basis of IOT technology, which is to monitor the main factors inducing smoldering, such as temperature, humidity, etc. and to monitor the changing environmental factors induced by cotton smoldering, such as carbon monoxide, smoke, and the like. Finally, the collected data would be analyzed comprehensively by the fusion algorithm of multi-sensor information in the application layer so as to realize the double early warning of cotton smoldering and warehousing fire triggered by smoldering, which is expected to avoid the cotton warehousing fire to the greatest extent.
\end{abstract}

Keywords: IOT, cotton warehousing fire, smoldering, early warning

\section{Introduction}

IOT technology has been used increasingly in the field of modern fire prevention and control because of its comprehensive perception, reliable transmission, intelligent analysis and so on. Guan Guocong (2012) applied the technology of distributed optical fiber temperature detection to solve complex environmental problems of strong electromagnetic interference in the research process of the fire alarm system of subway tunnel, which realized the fire monitoring in complex environment[1]. Shu Shi(2015) built up the simulation control center based on the IOT technology of short-range wireless communication in the research of detection system of bus blaze, which improved the function and operating efficiency of the original system[2]. Sadat (2015) and the fellows developed a warning system of forest fire by the remote sensing technology of satellite to achieve the effective alarm and accurate positioning of forest fire[3]. According to the above mentioned, the IOT technology has been effectively applied to the fire prevention and control, and also has made great contribution to the fire prevention and control. However, according to our careful analysis we found that there was "early warning" statement in the existing researches on the fire prevention and control, which should be classified as "warning" instead of "early warning" because action was taken only after catching fire.

Application of IOT technology to the prevention and control of cotton fire is still in the primary stage, and there are only a few research findings. Zhu Qixiang, Wu Guoxin and the fellows applied smoke detector, temperature detector and optical detector to the alarm system in the research of fire alarm technology of cotton warehousing, which enhanced the environmental monitoring capability of cotton storage and improved its performance and accuracy. While the fire sensor network with cable technology has poor expansibility and complicated wiring, and the aging and damage of lines would result in high rates of alarm failures or errors[4]. In their follow-up studies, ZigBee technology was applied to 
the fire alarm system, which has made up for the shortcomings of the wired network and had the following advantages of low cost, low power consumption, flexible networking, short waiting time, simple operation, wide coverage, but this scheme made early warning only after catching fire, and the alarm was mainly based on the single index[5]. Since it is really difficult to put out cotton fire and burned cotton may lose its value, the alarming after fire would still cause huge losses. Therefore, only the effective early warning of cotton fires can ensure the safe storage of cotton. In order to realize the effective early warning of the cotton fire, it is essential to find out the formation mechanism of the cotton fire. Gu Lihua found that smoldering was the main cause of cotton fire apart from open fire through comprehensive analysis of the causes of cotton fire from the physical and chemical properties and combustion condition of cotton, and proved with experiments that the temperature and humidity were the important factors affecting cotton smoldering and that cotton smoldering would produce some smoke[6]. Xia Enliang made a comparative study of combustion characteristics between cotton burning and smoldering and found that the smoldering process would release more carbon monoxide and the average generation rate of $\mathrm{CO}$ was higher than burning[7]. Wang Jinjun found that cotton smoldering was influenced by environment temperature and humidity, and it would also produce carbon monoxide and smoke in his research[8]. From the above characteristics of cotton smoldering, it can be seen that the research on the formation mechanism of smoldering is not sufficient, while the influencing factors of smoldering and its impact on the environment is almost clear, which provides valuable theoretical support for us to study the problem of fire early warning.

Therefore, we can learn from the existing research results of prevention and control of cotton fire, make deep analysis of the formation mechanism of cotton smoldering, collect all related information of cotton storage environment with the application of IOT technology, and research the collected data comprehensively through scientific algorithm, so as to achieve effective early warnings of cotton smoldering and warehouse fire caused by the smoldering and thus avoid the cotton warehousing fire to the greatest extent.

\section{Analysis on the Formation Mechanism of Cotton Fire}

Cotton has special physical and chemical characteristics of inflammability, smoldering, spontaneous combustion. In order to achieve the effective prevention and control of cotton fires and to ensure the safety of cotton storage, it is essential to know accurately the physical and chemical characteristics of cotton and understand deeply the formation mechanism of cotton fires.

\subsection{Physical and Chemical Characteristics of Cotton}

The main components of cotton fiber include cellulose, fat, wax and other flammable substances. Cellulose has a large number of hydrophilic hydroxyl groups, and can be expanded in water. Cotton fiber can be weakened under the long action of water vapor, which is then oxidized by the oxygen in the water vapor and can give out heat. There is a certain gap between the cotton fiber molecules, and the gap can absorb moisture from the air or release moisture into the air. This performance is referred to as moisture absorption. At a fixed temperature, the greater the relative humidity is, the more the amount of moisture will be, and the more the amount of heat will be released. In addition, some scholars estimate that the total gaps inside or between the cotton fibers account for about $20 \%-40 \%$ of the entire volume. Under the stuffy condition of the pores, the heat conduction is slow, which provides heat storage and preservation. The small amount of air of the pores in the cotton fibers can maintain a slow burning of the local or small area of the cotton in the case of insufficient external oxygen. 


\subsection{Model Construction Based on the Condition of Cotton Spontaneous Combustion}

Generally, only when the heat generated inside is more than that emitting outside, can the spontaneous combustion of cotton be triggered. Such condition can be formed in the following two aspects. On the one hand, the unit mass of cotton will produce a lot of heat because of the heat released by moisture absorption, by fermentation, by oxidation, etc.; on the other hand, because of the numerous pores of cotton fiber, the heat conduction coefficient of cotton is very small, providing strong heat preservation. At the same time, the cotton usually stores in packs, and the internal heat of cotton bags in the lower stacking can hardly be released. Furthermore, the poor ventilation of cotton warehouse also causes the stuffy air inside the cotton bag, resulting in heat accumulation.

The spontaneous combustion of cotton refers to the burning phenomenon under normal pressure and temperature caused by the heat accumulation of the chemical reaction, biological function, or physical change instead of open fire. In the process of cotton warehousing, the increasingly accumulated heat inside the cotton stacking results in spontaneous combustion of cotton, which also indicates some typical signs: heating and smoking before open fire, and white smoking first, and then black smoking. The tight bundling of the cotton causes the lack of oxygen inside the cotton stacking, which maintains the status of smoldering. When the smoldering cotton suddenly encounters air convection, the incomplete combustion of cotton quickly becomes a complete combustion, and also the flameless combustion quickly turns into flaming. Large amounts of carbon monoxide may be produced under the condition of long smoldering. While the ignition point of carbon monoxide is $12.5 \%-74 \%$, it is extremely easy to generate deflagration with air mixture, causing cotton warehouse fire.

According to the above analysis, we construct a conceptual model of cotton smoldering, which is shown in Figure 1.

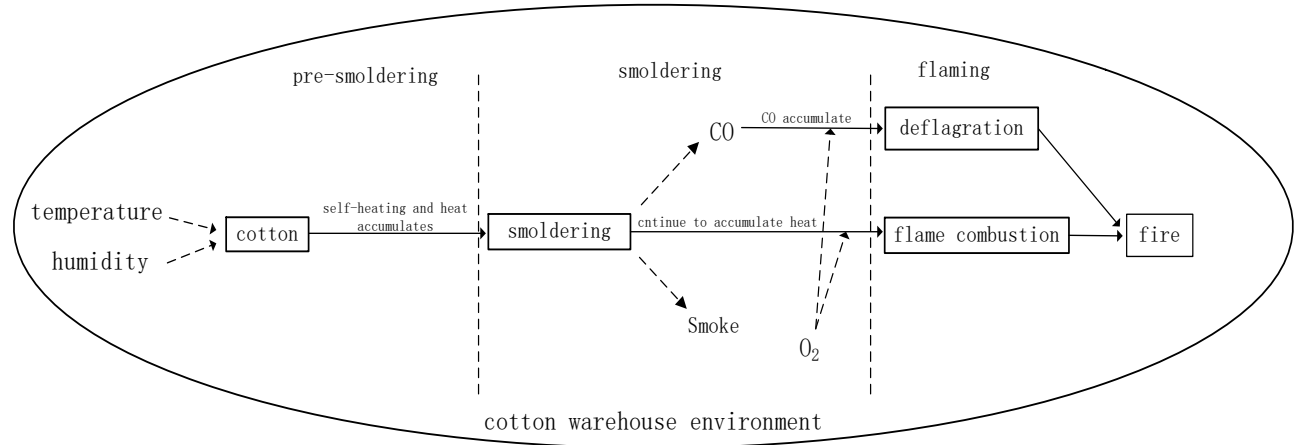

Figure 1. Conceptual Model of Cotton Smoldering

The model divides cotton smoldering into three stages: the first stage is pre-smoldering. Due to the external environment and self-heating of cotton bag, the heat accumulates and gradually develops to the burning conditions of smoldering. The second stage is smoldering. The increasingly accumulated heat eventually results in spontaneous combustion of cotton, releasing $\mathrm{CO}$ and smoke gradually. At the same time, the tight bundling of the cotton causes the lack of oxygen inside the cotton stacking, which maintains the status of smoldering. The third stage is the cotton warehouse fire caused by open fire. The causes can be analyzed from two aspects: (1) with smoldering continued, the heat continues to accumulate, when the smoldering cotton suddenly encounters air convection, the incomplete burning of cotton quickly becomes a complete burning, and the flameless combustion is rapidly converted to the flaming, causing fire of cotton warehouse. (2) The concentration of $\mathrm{CO}$ increases gradually with the smoldering process. When the concentration reaches the deflagration limit, $\mathrm{CO}$ will explode with air mixture, causing the cotton warehouse fire. 
Although the causes of cotton smoldering may not be limited to the current knowledge, the early warning of cotton smoldering is feasible in theory according to the current research findings. The qualitative research was only employed in the analysis of causes of cotton smoldering, but the main influencing factors of cotton smoldering and the changing environmental factors caused by smoldering can also be figured out through the above analysis. The real-time characteristic information of these factors can be obtained through the corresponding collecting devices, which provides the basis for judging the probability of fire. With the strengthening of formation mechanism research of cotton smoldering and deep understanding of the corresponding influencing factors and their logical relationship, the validity of the model will also be improved continuously.

\section{Design of Cotton Fire Early Warning Scheme}

\subsection{Internet of Things Technology}

\subsubsection{Internet of Things}

Internet of Things is "the Internet of connecting objects". "Internet of Things concept" is network concept which is to extend clients to any items on the basis of "Internet concept", in order to exchange and communicate information. One of the most widely accepted definitions is the description given by the International Telecommunications Union (ITU) in 2005. The Internet of Things is a network that in accordance with agreements, connects any items with the internet, exchange and communicate information by radio frequency identification (RFID), infrared sensors, Global Positioning System (GPS), Laser scanner and other information sensing equipment to achieve the intelligent identification of goods, positioning, tracking, monitoring and managing of objects [9].

\subsubsection{Internet of Things System Architecture}

From the technical point of view, the Internet of things can be divided into three layers: the perception layer, the network layer, and the application layer. The perception layer is responsible for data acquisition and conversion, includes the general sensor nodes which forms the wireless sensor networks, the sensing gateway node which connects wireless sensor networks, mobile communication networks and the Internet. Different Sensors arranged in the perception layer can be fully aware the surrounding environment, identify the current environment condition, and transfer outward. The current sensor technology is very mature. The sensors that can be used for fire early warning are light sensor, temperature sensor, smoke concentration sensor. They can fully collect data, detect fire and send fire information for the cotton warehouse.

The Network layer is responsible for data transmission and processing. The network communication infrastructure includes mobile communication networks and the Internet. Collected information can be transferred reliably through the network layer. Bluetooth, GPRS, 3G and other communication technology provides guarantee for the reliable information transmission. These communication technologies can quickly transfer information like data, images, sound, and video, which is the detailed and intuitive information support for the fire automatic warning system.

The Application layer is responsible for data management and application, mainly includes a variety of network of things applications, such as fire monitoring, environmental monitoring, intelligent transportation, telemedicine, intelligent city management and industrial monitoring. The Application layer is the core value of the Internet of things technology. It is aim at processing collected perceptual information intelligently and providing customized information services for clients. The application layer can intelligently analyze the data from various sensors in the cotton warehouse to judge whether there is a fire or not, and provide the fire rescue plan quickly. 
According to the characteristics of the three aspects of the Internet of Things technology, applying the technology of Internet of Things to the fire early warning system, can fully perceive the fire environment, monitor the reliable data transmission and intelligent analysis. It provides deep development for the fire early warning system and promotes the system's developing toward network, intelligent and high reliability.

\subsection{Overall Architecture Design}

Fire early warning system functions include fire data acquisition, information transmission and background analysis. In this paper, a fire early warning system project based on the Internet of Things technology is designed. The project forms the original stand-alone fire-fighting equipment to a self-organizing network (AD HOC) through ZigBee technology, then transmits various data information to the control center by GPRS network and Internet. The control center monitors and controls everything. The specific structure is presented in Figure 2

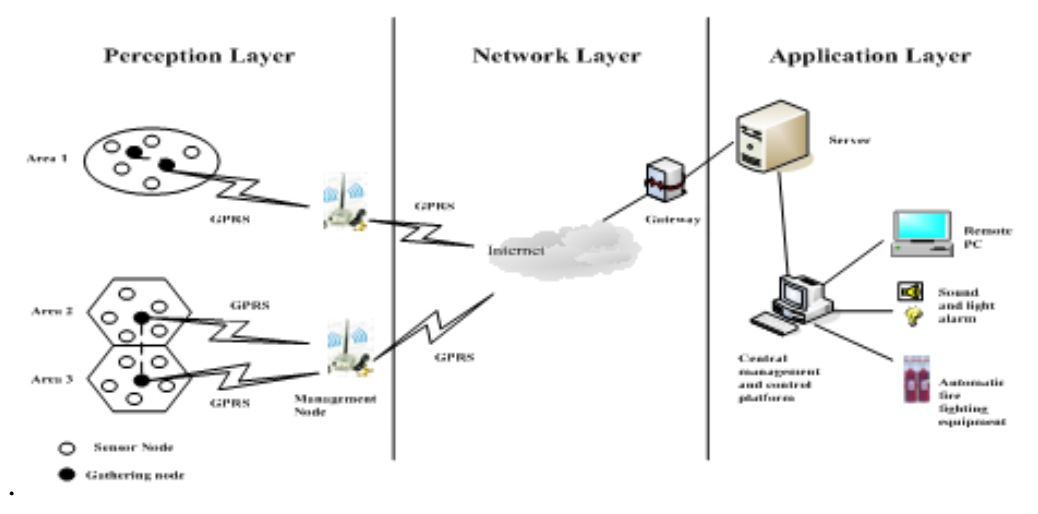

Figure 2. Architecture of Fire Early Warning System

The data collection network of the perception layer adopts the ZigBee tree network, and consists of sensor node, aggregation node and management node. When the system is deployed, the aggregation nodes are fixed in the monitored area. They are the routers in the unit area to collect network information. The sensor nodes can be randomly arranged according to actual needs. In this way, the sensor nodes will continue to send the environment-aware data to the aggregation node, and the aggregation node will send them to the management node. The management node stores the data to the network server via GPRS and Internet. The control center accesses the data on the network server, analyzes and processes the data collected from different areas and different sensors and intelligently judges fire according to the calculation model and the judgment criterion. The Control center starts early warning according to the possibility of fire, through the sound, photoelectric and other warning methods, then starts the automatic fire equipment, to achieve the effective control of the fire.

\subsection{Stratified Design of Fire Early Warning System}

\subsubsection{Perception Layer Design}

(1) Sensor selection: According to the current research results, it is clear that the formation of smoldering is closely related to the cotton warehouse environment temperature and humidity. At the same time, the smoldering process will produce carbon monoxide, smoke. Therefore, the front perception layer of the internet of things selects temperature, humidity, carbon monoxide concentration and smoke sensors in this paper.

(2) Sensor layout: The sensing device of the perception layer is the main source of collecting information in the fire early warning system based on Internet of Things 
technology. The sensor of the perception layer has ZigBee tree network layout and includes smoke sensor module, carbon monoxide concentration sensor module, temperature sensor module, data acquisition module and ZigBee wireless module. ZigBee technology is a two-way wireless communication technology which has close distance, low complexity, low power consumption, low data rate, low cost, flexible networking, easy use and wide coverage. According to the requirement of the fire early warning system, compared with Other wireless network technology, ZigBee technology is mainly applied to automatic control and remote control, is more suitable for the formation of a wide range of wireless fire detector network. For the ZigBee tree network structure, each master node can not only directly connect with the sensor for data acquisition and monitoring, but also automatically transfer data from other network nodes. In addition, each master node can be connected wirelessly with child nodes which do not assume the network information transfer tasks within their own signal coverage range. Cotton warehouse fire early warning system's ZigBee tree network structure is the guarantee for each monitoring cotton warehouse's environmental real-time data collection and transmission. cotton warehouse fire early warning system perception layer design scheme is presented in Figure 3.

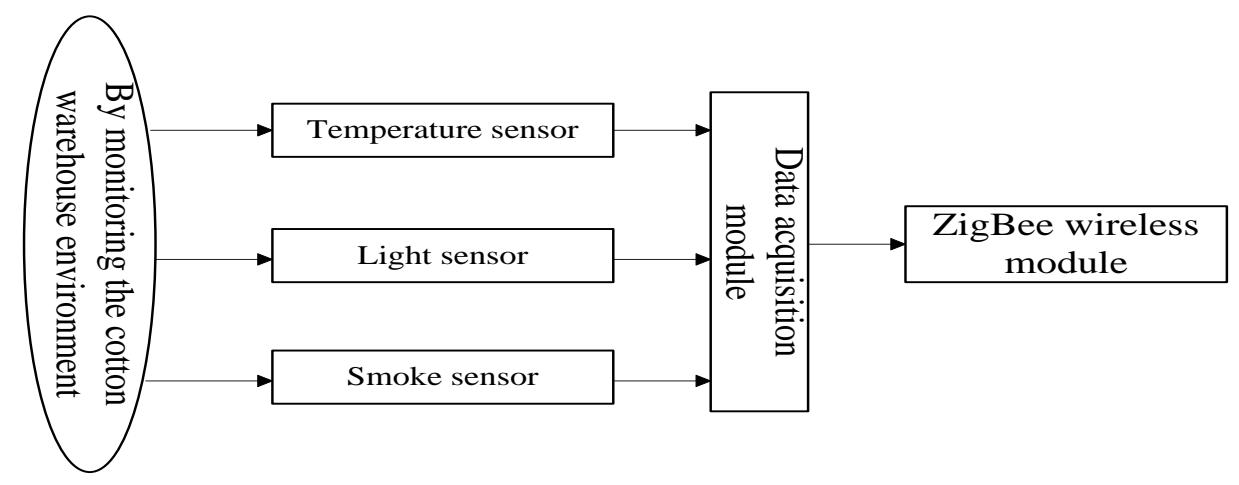

Figure 3. Perception Layer Design

\subsubsection{Network Layer Project Design}

The network layer of the cotton storage fire warning system mainly uses the wireless GPRS and the cable Internet for data transmission. The GPRS wireless transmission is widely used, and its technology is relatively mature. It has several advantages, such as the wide coverage of network signal, the low communication cost, Internet and the achievement of remote, real-time, uninterrupted and reliable high-speed data transmission. It is ideal for the need of the wireless data transmission network for the cotton storage fire warning system. Internet of Things is developed on the basis of the Internet, and they are inseparable. The collected data by the fire warning system will be stored on the server through the cable Internet transmission, and will be analyzed by the application layer. The general work flow of the network data transmission of the cotton storage fire warning system network layer is: The sink node first receives the data sent by the wireless sensor node, then transmits the data to the Internet through the wireless GPRS network and stores the data on the server which is connected with the Internet. Network Layer Work Flow is presented by Figure 4.

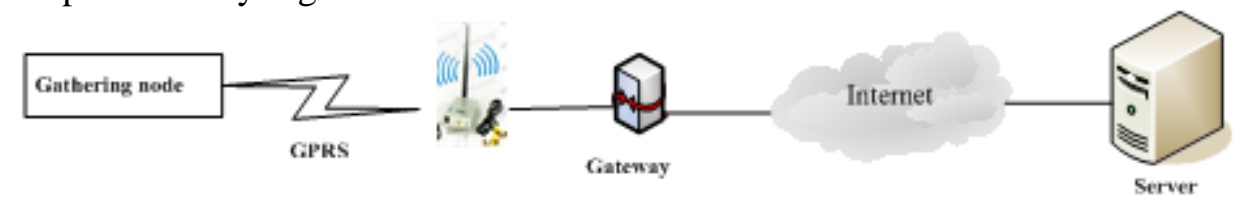

Figure 4. Network Layer Work Flow 


\subsubsection{Application Layer Project Design}

The control center obtains data through real-time access to the server, judges the source of data, decides whether the data exceeds the warning value, locates the data beyond the warning range, and starts the warning device. The Control Center Main Module is presented by Figure 5 .

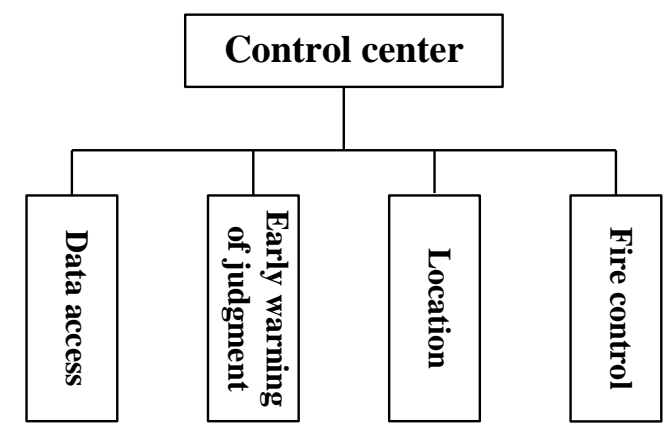

Figure 5. Control Center Main Module

The sensor network located in the cotton warehouse can collect real-time and accurate data, such as temperature, humidity, carbon monoxide concentration, and Smoke and upload them to the server. The control center carries on the intelligent Analysis to the cotton warehouse environment data information by visiting the server. The system will start the smoldering early warning if the temperature and humidity data fusion calculation results reach the smoldering early warning threshold. The system will start the cotton warehouse fire early warning (smoldering alarm) if the temperature, humidity, carbon monoxide concentration, and smoke data fusion calculation results reach the smoldering threshold. At the same time the system will quickly determine the anomaly point (fire point) by locating the sensor which generating the abnormal data on the electronic map and start the appropriate disposal plan.The Cotton Warehouse Fire Early Warning System Flow is presented by Figure 6.

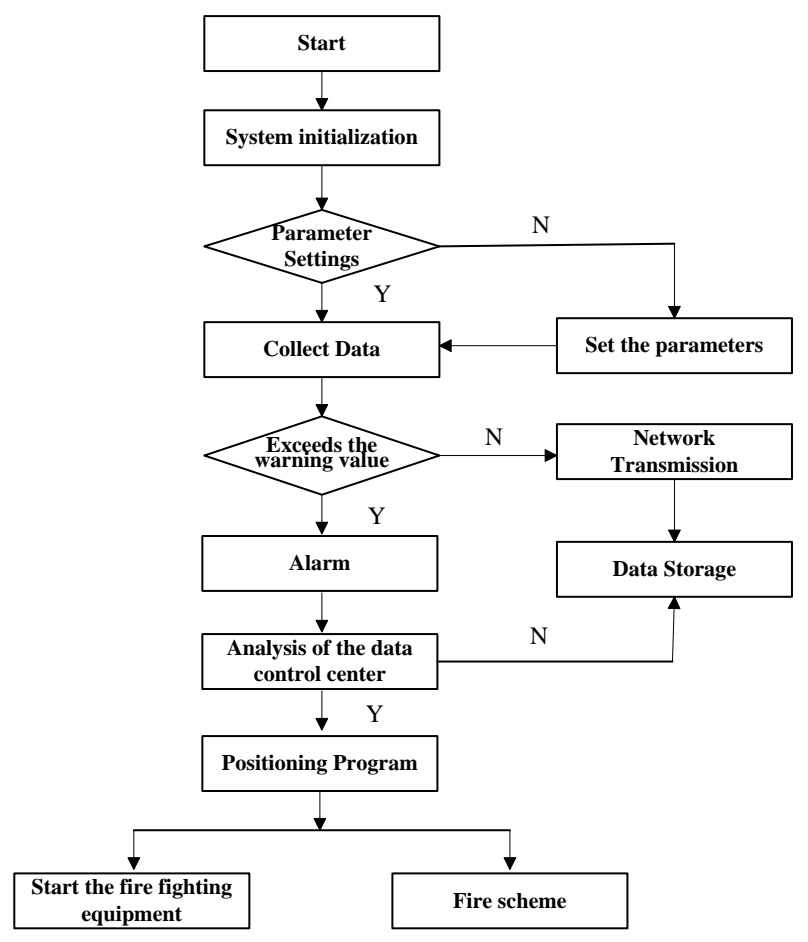

Figure 6. Cotton Warehouse Fire Early Warning System Flow 
The scientific cotton warehouse fire warning system architecture is formed and the cotton warehouse environment monitoring information comprehensive collection and the transmission safety are achieved through the rational design of the above hierarchical structure. However, in the whole cotton warehouse fire early warning system application plan design process, the keys of the smooth and effective implementation of the project are how to dispose the data that temperature, humidity, light and smoke sensors collect and how to analyze the early warning conditions through the data sensors gathered. This paper will use the fire early warning algorithm which is based on multi-sensor information fusion to solve the above problems effectively and provide technical support for the application of cotton warehouse fire early warning system.

\section{Multi Sensor Information Fusion Algorithm}

The fire early warning system this paper mentioned uses temperature sensor, humidity sensor, carbon monoxide concentration sensor and smoke sensor to collect information. However, the multi-sensors handle multiple signals simultaneously may bring redundancy and contradiction information. Therefore, it is urgent to further process the information, which can be achieved by controlling and using various sensors and their observation information reasonably, combining collected information by a certain optimization criterion, and producing the interpretation and description of the consistency of the observation environment. Therefore, the "Information fusion" is dealing with multi-sensor data extensively, that is, combining or integrating data which is from multiple sensors or other information sources to obtain a comprehensive estimate value of the cotton warehouse fire early warning.

\subsection{Fire early Warning Algorithm Analysis}

\subsubsection{Fundamental Principle}

Multi-sensor information fusion can also be called multi-sensor data fusion, or multisource information fusion, which refers to gaining the consistent interpretation and description for tested objects to achieve the appropriate decisions and estimates through the analysis, synthesis and applications of data from a variety of sensors in different time or space in accordance with certain criteria. Multi-sensor redundancy enhances the reliability of the system, and their complementary impairs the unreliability of single sensor. Multi-sensor information fusion enhances the reliability and credibility of information, which is much better than any other single sensor [10].

In fact, the multi-sensor information fusion is very similar to the function of the human brain handling the information collected from various organs (ears, nose, eyes, etc.). Brain will gather all kinds of information (taste, sight, sound, touch feeling, etc.), extract the information features according to prior knowledge, and then judge and estimate the environment and what is happening around. It could be said that the multi-sensor information fusion is a functional simulation of the human brain. It combines and analyzes all kinds of information from a variety of sources to give a consistent interpretation or description of the tested object.

\subsubsection{Process of Fusion}

Information fusion process includes multi-sensor information acquisition, information preprocessing, feature extraction, fusion computing and result recognition, as shown in Figure 7. Information acquisition refers to collecting information around, such as temperature, humidity, carbonmonoxide concentration, smoke and so on, and then by A/D converter, convert them to digital signals which are accessible for the microprocessor. Information preprocessing mainly means to filter wave bands and noise on the digital 
signal we obtained. At present, most of sensors integrated information acquisition and preprocessing, which make it possible to obtain accurate and useful digital signal directly from the output of the sensor. The temperature and humidity sensor tn this paper is one like that, which omits information preprocessing. Finishing feature extraction on preprocessed information, we do inference, estimates and fusion computing on the extracted characteristic for final recognition result.

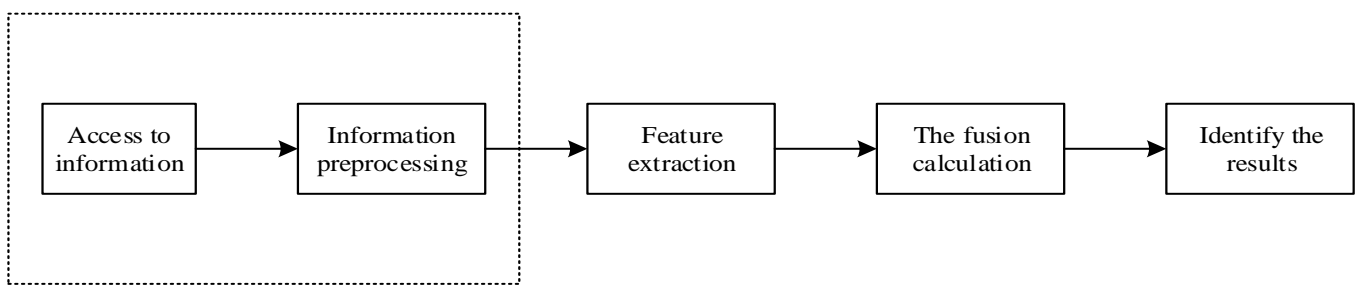

Figure 7. Steps of Information Fusion

\subsubsection{Hierarchy Structure}

According to different information levels on the sensor, multi-sensor information fusion can be divided into pixel fusion, feature fusion and decision fusion.

(1) Pixel fusion: Pixel fusion refers to fuse the data on sensors before preprocessing. that is to say to integrate and analyze the initial data. Its structure is in Figure 8. pixel fusion can save and provide more original information than the others. Algorithm of pixel fusion includes classic speculation and estimation theory. It is the minimum level fusion. Because of the huge volume of information, long processing, low instantaneity, and instability from original information, the system needs higher correction capability in the pixel fusion.

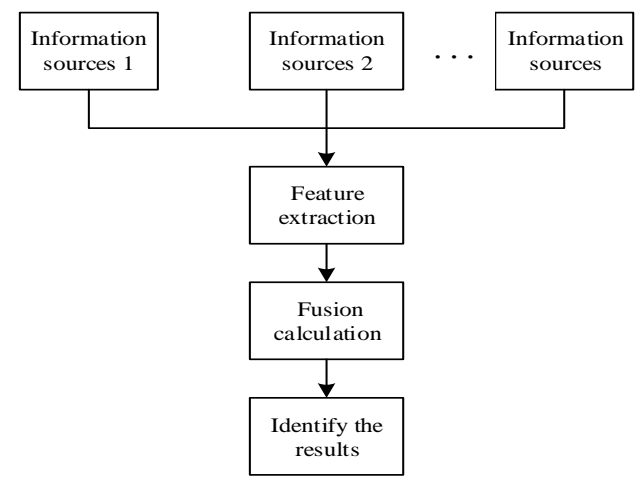

Figure 8. Pixel Fusion

(2) Feature fusion: Feature fusion belongs to the intermediate level, and its structure is shown in Figure 9. It refers to extracting feature from preprocessed data. It finished a statistic compressor of information from pixel fusion and makes classification, analysis and integration with feature information. The advantage of Feature fusion is to compress complex information volume, and to improve the processing instantaneity. Moreover, because the features can impair decision, the result of feature fusion on large extent contribute useful information on decision analysis. The disadvantage is the less accuracy compared with pixel fusion due to the data loss.

Feature fusion can be divided into two categories: target data feature-fusion and target data state-fusion. target data feature-fusion requires to process feature accordingly before the fusion and divides feature vectors into various different combinations. target data state-fusion is mainly applied in multi-sensor target-tracking field, and its mainly includes the data-matching, and parameters correlation and state vector estimation after successful matching. 


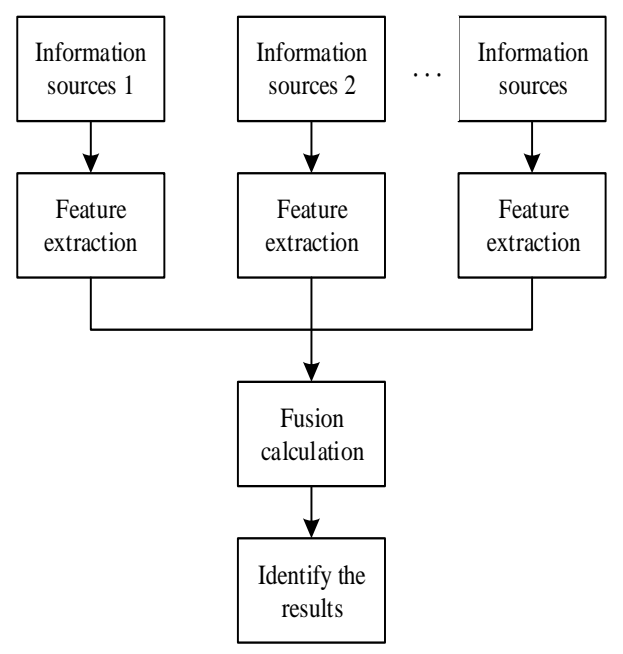

Figure 9. Feature Fusion

(3) Decision fusion: Compared with the former two fusions, decision fusion is higher, which refers to making the best decision based on completed decision of various information sources according to certain criteria. Its structure is shown in Figure 10. Because decision fusion result is regarded as the foundation of command and control, so we must make full use of feature information extracted from various feature-level sensor, and calculate the results by appropriate fusion technique. The results directly influence the decision level.

Main advantages of Decision fusion are: low requirements of bandwidth for transferring information, strong noise immunity ability; low processing cost of fusion center; a higher flexibility though one or more sensor fails, the system still can produce correct results through proper fusion.

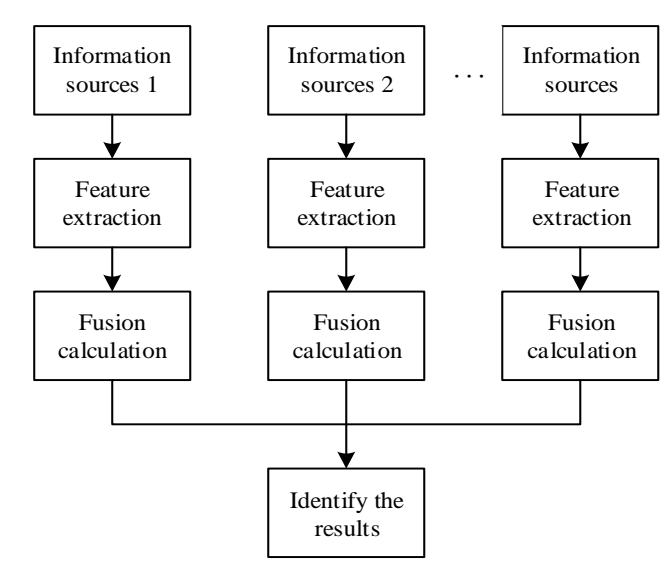

Figure 10. Decision Fusion

Advantages and disadvantages of the three fusions above are shown in Table 1. 
Table 1. Comparison of Advantages and Disadvantages of Three Fusions

\begin{tabular}{|l|l|l|l|l|l|l|l|l|l|l|}
\hline $\begin{array}{l}\text { Fusion } \\
\text { Model }\end{array}$ & $\begin{array}{l}\text { Calcul } \\
\text { ation } \\
\text { Volu } \\
\text { me }\end{array}$ & $\begin{array}{l}\text { Fault } \\
\text { Toler } \\
\text { ance }\end{array}$ & $\begin{array}{l}\text { Infor } \\
\text { matio } \\
\text { n Loss }\end{array}$ & $\begin{array}{l}\text { Acc } \\
\text { urac } \\
\text { y }\end{array}$ & $\begin{array}{l}\text { Noise } \\
\text { Immuni } \\
\text { ty }\end{array}$ & $\begin{array}{l}\text { Fusio } \\
\text { n } \\
\text { Meth } \\
\text { od }\end{array}$ & $\begin{array}{l}\text { Homog } \\
\text { eneity } \\
\text { Requir } \\
\text { ements } \\
\text { Of The } \\
\text { Sensor }\end{array}$ & $\begin{array}{l}\text { Comm } \\
\text { unicati } \\
\text { on } \\
\text { Data } \\
\text { Volum } \\
\text { e }\end{array}$ & $\begin{array}{l}\text { Instan } \\
\text { taneit } \\
\text { y }\end{array}$ & $\begin{array}{l}\text { Fusio } \\
\text { n } \\
\text { Level }\end{array}$ \\
\hline Pixel & Large & Bad & Small & High & Weak & $\begin{array}{l}\text { Diffi } \\
\text { cult }\end{array}$ & More & Large & Bad & Low \\
\hline Feature & $\begin{array}{l}\text { Mediu } \\
\text { m }\end{array}$ & $\begin{array}{l}\text { Medi } \\
\text { um }\end{array}$ & $\begin{array}{l}\text { Mediu } \\
\text { m }\end{array}$ & $\begin{array}{l}\text { Med } \\
\text { ium }\end{array}$ & Normal & $\begin{array}{l}\text { Norm } \\
\text { al }\end{array}$ & $\begin{array}{l}\text { Comm } \\
\text { on }\end{array}$ & $\begin{array}{l}\text { Mediu } \\
\text { m }\end{array}$ & $\begin{array}{l}\text { Com } \\
\text { mon }\end{array}$ & $\begin{array}{l}\text { Medi } \\
\text { um }\end{array}$ \\
\hline $\begin{array}{l}\text { Decisio } \\
\mathrm{n}\end{array}$ & Small & Good & Large & Low & Strong & Easy & Less & Small & Good & High \\
\hline
\end{tabular}

It is obvious that pixel fusion can provide micro-information of the target. Because the large volume of information, its characteristics is high processing cost, large communication data volume and strong noise immunity. Decision fusion is with low bandwidth requirements for transferring information, strong noise immunity and low processing cost of fusion center, and higher flexibility when one or more sensor fails, the system still can produce correct results through proper fusion. While the fire warning system needs stronger noise immunity and better instantaneity, therefore the Decision fusion is best choice.

\subsubsection{Algorithm of Fuzzy Neural Network Fusion}

In terms of the screening result of the various algorithms mentioned in Chapter 1, here the author would adopt the algorithm of fuzzy neural network fusion to solve the problems. Because the data collected from multi-sensor are unstable, we take real number between 0 and 1 to represent the truth of the information collected by the sensors, which constitutes fuzzy sets of the system. According to fuzzy rules, we make a comprehensively reason of fuzzy sets and environment information and get a decision with high reliability. The flow chart of algorithm of fuzzy neural network fusion is shown in Figure 11. the process is as follows:

1) Select $N$ sensors to collect environment information;

2) Make feature extraction of collected information;

3) Make normalization of feature signal as input signal of neural network;

4) set Normalization signal and known state as training samples and train the neural network to achieve reasonable weight coefficient. Finishing the training, an we can get a output state as long as inputting normalization multi-sensor feature information into neural network.

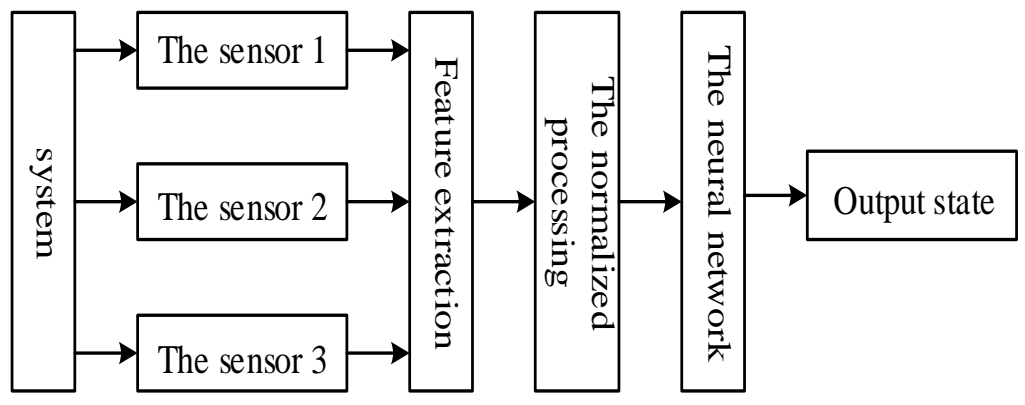

Figure 11. Procedure of Fuzzy Neural Network Fusion 


\subsection{Fire Algorithm Design with Multi-sensor Information}

(1) Extraction of Feature Information

In this paper, information sources include temperature, humidity, carbonmonoxide concentration, smoke sensors. When firing, the indoor temperature, humidity, carbonmonoxide and smoke concentration will not rise soon. They change slowly until the fire become worse to some extent, simple information reach traditional alarm threshold. While at that time the fire is out of control. we can gather the changing data of temperature, humidity, carbonmonoxide and smoke concentration, and calculate the tendency of their relative changes.

We collect consecutive information from six temperature sensor, setting it as a vector $V=\left[V_{1}, V_{2}, V_{3}, V_{4}, V_{5}, V_{6}\right]$, the difference between statistical vector elements can be shown as formula (1)

$$
\text { Diff }=\left|V_{1}-V_{2}\right|+\left|V_{3}-V_{4}\right|+\left|V_{5}-V_{6}\right|
$$

We set $\mathrm{V}_{\text {thread, }}$ a threshold of the temperature. The characteristic information of temperature, it is to say the credibility which means fire, is marked as the formula (2). The characteristic information calculation of the temperature, humidity and carbonmonoxide concentrationt is similar with that of the temperature.

$$
F=\left\{\begin{array}{cl}
1 & \text { if } \quad \text { Diff }>V_{\text {thread }} \\
\frac{\text { Diff }}{V_{\text {thread }}} & \text { else }
\end{array}\right.
$$

For the characteristic information of smoke, when smoking, we set its characteristic value as 1 , no smoke, the value is 0 .

(2) Fuzzy reasoning fusion

We assume that A is a set of decision in system, and that B is a set of information source, and that the element aij in the matrix $\mathrm{PA}^{*} \mathrm{~B}$ is the subjection degree, the possibility that information source $i$ could be speculated into result $j$. We set $X$ as the credibility of all information source, through fuzzy transformation of $\mathrm{Y}=\mathrm{X} * \mathrm{PA} * \mathrm{~B}$, the result $\mathrm{Y}$ is regarded as the possibility of decisions.

For a variety of possible decision results, we should make judgment in accordance with the following judging criteria:

1) There must be a maximum value in various kinds of decision possibility;

2) The possibility of judgment result must be greater than certain threshold;

3) The possibility of judgment result must be over the certain threshold than any other decision probabilities.

In this system, the set $\mathrm{X}$ represents the credibility of all information source as shown in formula(3) .

$$
X=\left[X_{1}, \quad X_{2}, \quad X_{3}, X_{4}\right]
$$

Matrix $\mathrm{P}$ is shown in formula(4), the first number in the first column means the subjection degree of all information source that there is fire. And we can calculate them by the formula (2). The second column represents subjection degree of all information source that there is no fire.

$$
P=\left[\begin{array}{ll}
a_{11} & a_{12} \\
a_{21} & a_{22} \\
a_{31} & a_{32} \\
a_{41} & a_{42}
\end{array}\right]
$$

The results $\mathrm{Y}$ of Fuzzy reasoning contains two elements, one is the possibility of a fire after fusion, another is the possibility of no fire after fusion. Algorithm chart of multisensor information fusion executed in the monitoring center is shown in Figure 12. 


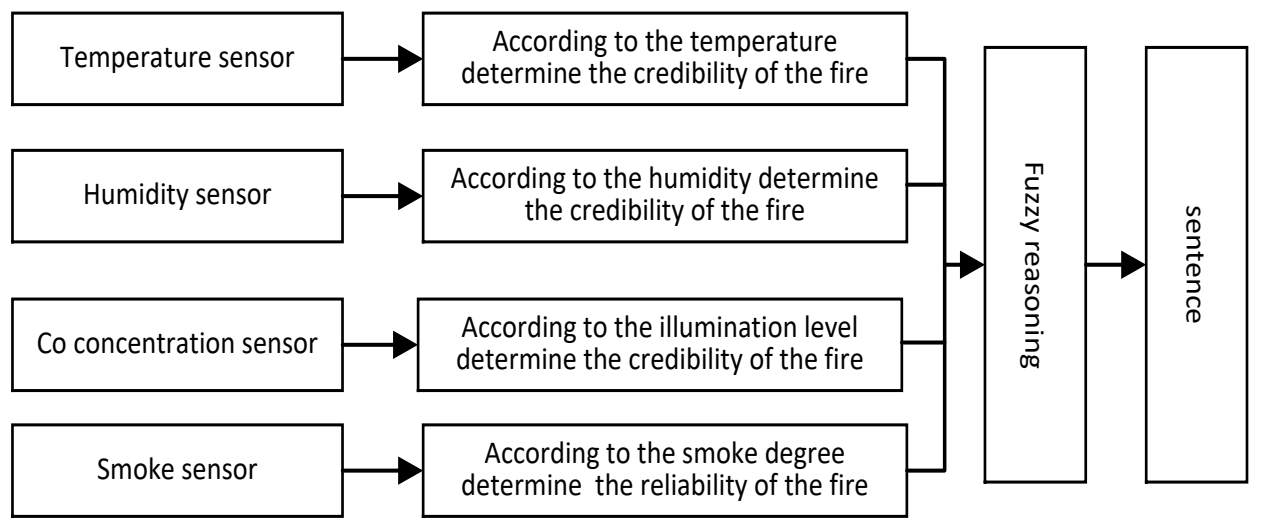

Figure 12. Algorithm Rocedure of Multi-Sensor Information Fusion

(3) The Fire detection test in algorithm of multi-sensor information fusion

During the test, for the first level warning, the warning of cotton smoldering, firstly we should set the credibility of judging whether there is smoldering, like the matrix X1. We set temperature credibility as 0.65 , humidity credibility as 0.35 . Because of no carbonmonoxide concentration and smoke before smoldering, so in the first level warning we can take the two factors out of consideration. For the second level warning, the warning when the cotton has been smoldering, we should set the credibility of judging whether there is fire, like the matrix $\mathrm{X} 2$. We set temperature and humidity credibility as 0.2 , carbonmonoxide concentration 0.31 and smoke 0.29 . In the system designed for this project, the smoke sensor only provides two kinds of signals, one is no smoke signal, another is the smoke signal. so the subjection degree of smoke judging if fire or not only can be 0 or 1 . The logistic of double warning is shown in Figure 12.

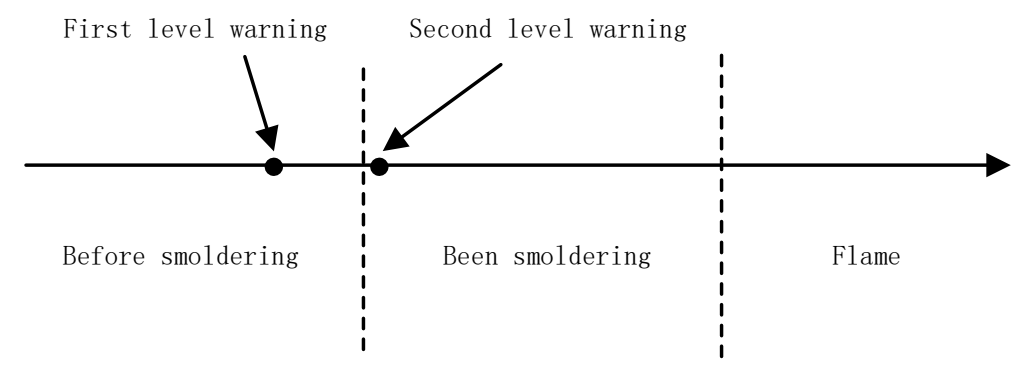

Figure 12. Logistic of Double Warning

If at some point, we set the subjection degree of temperature judging firing is 0.45 , the subjection degree of temperature judging no fire is 0.55 ; the subjection degree of humidity judging firing is 0.55 , the one judging no fire is 0.45 ; the subjection degree of carbonmonoxide concentrationt judging firing is 0.7 , the one of no fire is 0.3 ; the subjection degree of smoke judging firing is 1 , the one of no fire is 0 . Through Fuzzy reasoning, the result $\mathrm{Y}$ is shown in formula (5).

$$
Y=\left[\begin{array}{llll}
0.2 & 0.2 & 0.31 & 0.29
\end{array}\right] *\left[\begin{array}{cc}
0.45 & 0.55 \\
0.62 & 0.68 \\
0.79 & 0.21 \\
1 & 0
\end{array}\right]=\left[\begin{array}{lll}
0.7489 & 0.2511
\end{array}\right]
$$

Through Fuzzy reasoning, according to the result Y, the possibility of fire is 0.7489 , and the possibility of no fire is 0.2511 . Assuming the decision threshold as 0.7 , according to the judgment criterion, we could judge there is a fire and send the 
warning signal.

In order to verify the validity and accuracy of the fire warning system, this paper simulates the real environment by controlling the temperature, humidity, carbonmonoxide and smoke concentration in the monitoring site. There is fifty simulation experiments results as follows.

Table 2. Experiments

\begin{tabular}{|c|c|c|c|c|c|c|c|c|}
\hline \multirow{2}{*}{$\begin{array}{l}\text { Subjection } \\
\text { Degree } \\
\text { Information } \\
\text { Sources }\end{array}$} & \multicolumn{2}{|c|}{ Experiment 1} & \multicolumn{2}{|c|}{ Experiment 2} & \multicolumn{2}{|c|}{ Experiment 3} & \multicolumn{2}{|c|}{ Experiment 4} \\
\hline & Fire & No fire & Fire & No fire & Fire & No fire & Fire & No fire \\
\hline Temperature & 0.81 & 0.29 & 0.45 & 0.55 & 1 & 0 & 0.36 & 0.64 \\
\hline Humidity & 0.70 & 0.30 & 0.72 & 0.28 & 0.51 & 0.49 & 0.47 & 0.53 \\
\hline $\begin{array}{l}\text { Carbonmonoxide } \\
\text { Concentration }\end{array}$ & 0.75 & 0.25 & 0.66 & 0.34 & 0.29 & 0.71 & 0.24 & 0.76 \\
\hline Smoke & 1 & 0 & 1 & 0 & 0 & 1 & 0 & 1 \\
\hline \multirow{2}{*}{$\begin{array}{l}\text { Subjection } \\
\text { Degree } \\
\text { Information } \\
\text { Sources }\end{array}$} & \multicolumn{2}{|l|}{$\ldots \ldots$} & \multicolumn{2}{|c|}{ Experiment 48} & \multicolumn{2}{|c|}{ Experiment 49} & \multicolumn{2}{|c|}{ Experiment 50} \\
\hline & Fire & No fire & Fire & No fire & Fire & No fire & Fire & No fire \\
\hline Temperature & $\ldots \ldots$. & $\ldots \ldots .$. & 0.72 & 0.28 & 0.61 & 039 & 0.76 & 0.24 \\
\hline Humidity & $\ldots \ldots$. & $\ldots \ldots$. & 0.77 & 0.23 & 0.36 & 0.64 & 0.67 & 0.23 \\
\hline $\begin{array}{l}\text { Carbonmonoxide } \\
\text { Concentration }\end{array}$ & $\ldots \ldots$. & $\ldots \ldots$. & 0.43 & 0.57 & 0.47 & 0.52 & 0.84 & 0.16 \\
\hline Smoke & $\ldots \ldots$. & $\ldots \ldots$. & 1 & 0 & 1 & 0 & 0 & 1 \\
\hline
\end{tabular}

Table 3. Results of Experiments

\begin{tabular}{|l|l|l|l|l|}
\hline \multirow{2}{*}{$\begin{array}{c}\text { Experiment } \\
\text { After Fusion) }\end{array}$} & \multicolumn{2}{|c|}{ Subjection Degree } & \multirow{2}{*}{$\begin{array}{l}\text { Judgment Results } \\
\text { From the System }\end{array}$} & \\
\cline { 2 - 3 } & Fire & No Fire & & \\
\hline Experiment 1 & 0.825 & 0.175 & Fire & Fire \\
\hline Experiment 2 & 0.729 & 0.271 & Fire & Fire \\
\hline Experiment 3 & 0.392 & 0.608 & No Fire & No Fire \\
\hline Experiment 4 & 0.240 & 0.760 & No Fire & No Fire \\
\hline$\ldots$ & $\ldots$ & $\ldots$ & $\ldots$ & $\ldots$ \\
\hline Experiment 48 & 0.721 & 0.279 & Fire & Fire \\
\hline Experiment 49 & 0.630 & 0.370 & No Fire & No Fire \\
\hline Experiment 50 & 0.526 & 0.424 & No Fire & No Fire \\
\hline
\end{tabular}

The simulation experiment results certify that the warning accuracy rate of this system is $100 \%$, which means that this system can rapidly and accurately discern the environment changes and make fire warning. At the same time, the different warning results hint the difference of fire tendency and speed. The results of data fusion close to the threshold more, the fire tendency is more obvious and it speed faster. Besides, according to warning results, we can judge the firing impact Dn of different factor ( $D_{n}=A \cdot X, A$ refers to fire subjection degree, $X$ is credibility), further distinguish the main factors to fire, and judge the fire precaution priority according to $D_{n}$. When making sure the main factor to fire, we can deal with this factor and make oriented fire prevention and control. For example, $D_{1}>D_{2}$, the impact factor priority is temperature > humidity, therefore we should cool the cotton warehouse, which could help to avoid wrong action that resulted in fire and to provide oriented prevention, further reduce the fire possibility and improve the prevention and control of fire in cotton warehouse. 


\section{Conclusion}

In this paper, the conceptual model of cotton smoldering was put forward for the first time based on the current research results of formation mechanism of cotton smoldering. In view of the main inducing factors of cotton smoldering, such as temperature, humidity, etc., and the changing environmental factors induced by smoldering, such as carbon monoxide, smoke and the like, we established a frontend monitoring network of cotton warehousing environment in accordance with the IOT technology architecture. And we also designed the data transmission scheme in the network layer, and applied the fusion algorithm of multi-sensor information to the comprehensive analysis of the data collected in the process, which makes up the shortcomings of traditional single index for judging the occurrence trend of cotton fire and objectively realizes the double early warnings of the cotton smoldering and the cotton warehouse fire triggered by smoldering. This has turned the level of alarm up to the level of early warning for the prevention and control of cotton fire, and therefore it will avoid the cotton warehousing fire to the greatest extent. Although the causes of cotton smoldering may not be limited to the current knowledge and even qualitative research was only employed in the analysis of causes of cotton smoldering, the main influencing factors of cotton smoldering and the changing environmental factors caused by smoldering can be figured out through the current research findings. The real-time characteristic information of these factors can be obtained through the corresponding collecting devices, which provides the basis for judging the probability of fire. Therefore, the model constructed based on the current research findings and the early warning scheme of cotton smoldering based on IOT technology are feasible in theory. With the strengthening of formation mechanism research of cotton smoldering and deep understanding of the corresponding influencing factors and their logical relationship, the validity of the model will also be improved continuously. At the same time, the implementation of this program will accumulate a large amount of data, providing the basis of information support for the follow-up study on the formation mechanism of cotton smoldering.

\section{References}

[1] G. C. Guan. the Application of Distributed Optical Fiber Temperature Measurement of Fire Warning System in Subway. Modern Business Trade Industry, vol. 24, no. 12, (2012), pp. 178-179.

[2] Shi S. (2015) Bus Spontaneous-Ignition Monitoring and Warning System Based on Data Fusion. Zhejiang University of Technology, Hangzhou.

[3] Z. Sadat, P. H. Reza and M. Rossi. Forest Fire Susceptibility Mapping in the Minudasht Forests, Golestan Province, Iran. Environmental Earth Sciences, vol. 73, no. 04, (2015), pp. 1515-1533.

[4] Q. X. Zhu, G. X. Wu and S. X. Xu. Application of Fire Alarm Technology in Cotton Storage Security. China Cotton Processing, vol. 11, no. 05,(2011), PP. 20-21.

[5] Q. X. Zhu, S. X. Xu and G. X. Wu. Application of Zigbee Technology to Automatic Fire Alarm System in Cotton Warehouse. China Cotton Processing, vol. 13, no. 06, (2011), PP. 19-22.

[6] L. H. Gu. Cotton the Cause of Spontaneous Combustion. Fire, vol. 23, no. 02, (2012), PP. 32-33.

[7] E. L. Xia. Study on the Characteristic Comparative of Cotton Smoldering And Flame Combustion. Fire Safety Science, vol. 22, no. 02, (2013), PP. 70-76.

[8] J. J. Wang, Y. Zeng and W. Yuan. Research on Early Characteristics of Cotton-heap Smoldering and Composite Detection of Active Aspirating in Cotton Warehouse. Journal of Safety and Environment, no. 02, (2012), PP. 205-208.

[9] J. Jiang, D. H. Yang and Z Gao. Study on Application of IOT in the Cotton Warehousing Environment. International Journal of Gridand Distributed Computing, vol. 8, no. 04, (2015), PP. 91-104.

[10] J. Zhang, Y. h. Lu and G. Lei. Intellective Fire Alarm System Based on Multi- Sensor Data Fusion. Computer Engineering and Applications, vol. 6, no. 02, (2006), PP. 206-212. 


\begin{abstract}
Authors
Jia Jiang, master's tutor, is the head of Logistics Engineering Department and the director of Modern Logistics and Supply Chain Management Institute of Hebei University of Science and Technology. He has worked in the university since 1989. He is also the tobacco logistics expert of State Tobacco Monopoly Administration, the advisory expert of China International Engineering Consulting Corporation. He teaches Logistics, Supply Chain Management, Transportation and Packaging for graduate students and undergraduate students. He has finished many research programs in the national and provincial level, e.g. the National Science-supporting program. He has published many articles on International meetings and important journals as LISS、ICMLC、 Computer Science, etc.
\end{abstract}
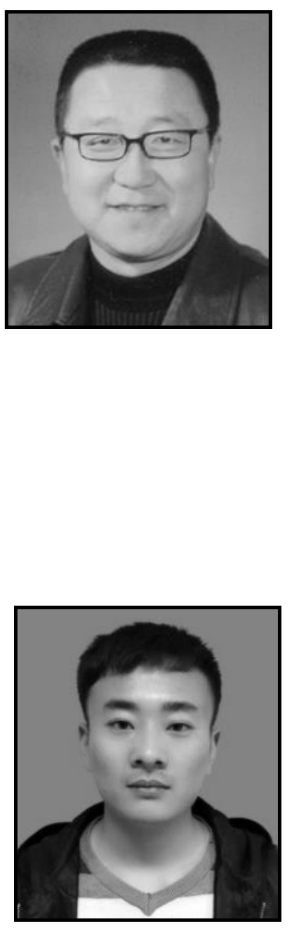

Damin Liu, he is a postgraduate student of Hebei University of Science and Technology. He is in the research of Logistics Service System Optimization and internet of things.

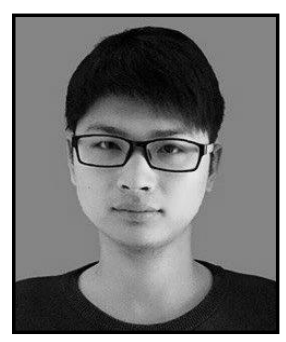

Yibo Jin, he is a postgraduate student of Hebei University of Science and Technology. He is in the research of Logistics Service System Optimization and internet of things. 